\title{
Onset of Glycemic and Weight Outcomes in Patients Initiating Exenatide Once Weekly: The Relationship of Exenatide Exposure with Efficacy over the First 24 Weeks of Treatment
}

Thomas Blevins · James Ruggles · Elise Hardy

Received: March 18, 2016 / Published online: May 5, 2016

(C) The Author(s) 2016. This article is published with open access at Springerlink.com

\begin{abstract}
Introduction: Exenatide is gradually released from exenatide once weekly (QW) microspheres, and at steady state, consistently controls glycated hemoglobin (HbA1c) in patients with type 2 diabetes (T2D). This post hoc analysis examined the timing to onset of clinical responses and their correlations with exenatide concentrations after initiation of exenatide QW in patients with T2D.
\end{abstract}

Methods: Trial data were retrospectively analyzed to explore the early clinical responses to exenatide QW, including the relationship of exenatide concentration with its effects on efficacy [fasting plasma glucose (FPG), HbA1c, and body weight] and tolerability (nausea and vomiting). Exenatide QW efficacy and

Enhanced content To view enhanced content for this article go to http://www.medengine.com/Redeem/ 73C4F06070F9D834.

T. Blevins $(\bowtie)$

Texas Diabetes and Endocrinology, Austin, TX, USA

e-mail: tblevins@texasdiabetes.com

J. Ruggles

AstraZeneca, Fort Washington, PA, USA

E. Hardy

AstraZeneca, Gaithersburg, MD, USA tolerability data were from DURATION-5, a 24-week, randomized, comparator-controlled trial [intent-to-treat (ITT) population]. Exenatide concentrations were measured in a patient subset (pharmacokinetic population).

Results: In the ITT $(n=129) /$ pharmacokinetic $(n=72)$ populations, baseline FPG, HbA1c, and body weight were $173 / 173 \mathrm{mg} / \mathrm{dL}, 8.5 \% / 8.4 \%$, and $97 / 98 \mathrm{~kg}$, respectively. Exenatide concentrations gradually increased until reaching steady state at week 8 . By week 4 , the FPG reduction $(-32.4 \mathrm{mg} / \mathrm{dL})$ was $94 \%$ of the week 24 reduction $(-34.6 \mathrm{mg} / \mathrm{dL})$. Reductions in $\mathrm{HbA1c}$ began by week $4(-0.6 \%)$ and stabilized by week 14 (week 24: -1.6\%). Weight reduction at week 4 was $-0.7 \mathrm{~kg}$ and decreased further (week 24: $-2.3 \mathrm{~kg}$ ). Peak nausea $(7.2 \%)$ and vomiting (2.4\%) occurred at weeks 6-8, declining thereafter.

Conclusion: Clinically relevant responses to exenatide QW were evident by week 4, after exenatide concentration passed the therapeutic threshold but before steady state was achieved.

Trial Registration: ClinicalTrials.gov identifier: NCT00877890.

Funding: AstraZeneca. 
Keywords: Body weight; Exenatide once weekly; Fasting plasma glucose; HbA1c; Nausea

\section{INTRODUCTION}

Type 2 diabetes (T2D) is a chronic disease necessitating long-term, individualized treatment. Challenges associated with some treatments include weight gain, risk of hypoglycemia, gastrointestinal side effects, and rapid loss of glycemic control [1-3]. Other factors considered by physicians and patients may include mode of administration, frequency of dosing, and initial timing of therapeutic response, particularly for patients with symptomatic hyperglycemia.

The glucagon-like peptide-1 receptor agonist (GLP-1RA) exenatide improves glycemic control and reduces weight, with a low risk of hypoglycemia when taken without concomitant sulfonylureas or insulin, but an increased risk of gastrointestinal events [4]. The once weekly (QW) formulation has demonstrated sustained glycemic control and is dosed infrequently [5], but the initial rate of therapeutic response has been questioned. Exenatide acts through multiple mechanisms of action, including stimulating insulin secretion in a glucose-dependent manner, suppressing glucagon secretion in a glucose-dependent manner, slowing gastric emptying, reducing food intake, and promoting satiety [4]. Dispersing exenatide in poly-(D,L-lactide-co-glycolide [PLG]) polymer microspheres-a biodegradable polymerallows for the continuous release of exenatide with regular QW therapy [6]. Exenatide is primarily released through diffusion and erosion of the microspheres. With regular dosing, plasma concentrations of exenatide QW increase gradually to steady state in approximately $6-8$ weeks [7-10]; however, treatment response may begin earlier, when concentrations surpass the therapeutic threshold [9].

The clinical development program for exenatide QW consisted of six pivotal phase 3 studies: the Diabetes Therapy Utilization: Researching Changes in A1C, Weight and Other Factors through Intervention with Exenatide Once Weekly (DURATION) trials. Data from DURATION-5 were used in this post hoc analysis [11], in which exenatide concentrations and clinical responses were measured for up to 24 weeks.

This analysis examined the relationships between exenatide concentrations and clinical responses to inform physician and patient expectations of exenatide QW therapy for treatment of T2D.

\section{METHODS}

Details of the DURATION-5 (ClinicalTrials.gov identifier: NCT00877890) study design were previously published [11]. Briefly, patients with T2D aged $\geq 18$ years who were treated for $\geq 2$ months with diet and exercise alone or with a stable, maximally effective regimen of metformin, sulfonylurea, thiazolidinedione, or a combination of these medications and had a glycated hemoglobin (HbA1c) of 7.1-11.0\%, fasting plasma glucose (FPG) $<280 \mathrm{mg} / \mathrm{dL}$, and a body mass index of $25-45 \mathrm{~kg} / \mathrm{m}^{2}$ were included in the trial. Patients were randomized to receive exenatide QW $2.0 \mathrm{mg}$ subcutaneously (SC) for 24 weeks $(n=129)$ or exenatide twice daily (BID) $5 \mu \mathrm{g}$ SC for 4 weeks followed by exenatide BID $10 \mu \mathrm{g}$ SC for 20 weeks $(n=123)$. Only patients treated with exenatide QW were included in this post hoc analysis. This article is based on a previously conducted study and does 
not involve any new studies of human or animal subjects performed by any of the authors.

FPG, HbA1c, and body weight were measured at weeks 1 (body weight only), 4, 8, 14, 20, and 24 in the intent-to-treat (ITT) population consisting of all randomized patients who received $\geq 1$ dose of study medication.

Exenatide concentration was determined by a validated immunoenzymetric assay [9] in a subset of patients in the ITT population, which was designated the pharmacokinetic (PK)-evaluable population, at baseline, at weeks $4,8,14$, and 20 (prior to administration of the next dose of study medication), and at study termination (week 24).

Nausea and vomiting adverse events (AEs) were self-reported at all office visits and categorized according to the Medical Dictionary for Regulatory Activities (MedDRA) version 12.0 in the ITT population.

\section{Statistical Analyses}

Demographics and baseline characteristics were reported descriptively. Because exenatide concentrations were not normally distributed, exenatide concentration was calculated as the geometric mean \pm standard error (SE). FPG was reported in $\mathrm{mg} / \mathrm{dL}$ [conversion factor to SI units $(\mathrm{mmol} / \mathrm{L})=0.0555]$. The least-squares (LS) mean \pm SE changes from baseline for FPG and HbA1c were calculated using an analysis of variance model based on a general linear model including factors for treatment group, HbA1c stratum $(<9.0 \%$ or $\geq 9.0 \%)$, and concomitant sulfonylurea use at screening. The LS mean \pm SE change from baseline in body weight was analyzed using a general linear model including factors for treatment group, baseline HbA1c stratum, concomitant sulfonylurea use at screening, and baseline body weight. Missing post-baseline values up to week 24 were imputed using the last observation carried forward approach. The statistical analyses were performed using SAS version 9.2 (SAS Institute, Cary, NC, USA).

A subanalysis examined mean ( \pm standard deviation) changes from baseline in FPG, HbA1c, and body weight among patients who had nausea AEs versus those without nausea (ITT population).

\section{RESULTS}

Baseline demographics and characteristics were generally well-matched between patients in the ITT $(n=129)$ and PK-evaluable $(n=72)$ populations, with small differences in age and race (Table 1). In the ITT population, 20 patients (15.5\%) treated with exenatide QW withdrew during the study (withdrawal of consent, $n=6$; $\mathrm{AE}, n=6$; lost to follow-up, $n=5$; loss of glucose control, $n=3$ ) [11].

Mean exenatide concentrations with exenatide QW treatment gradually increased until reaching steady state at approximately 8 weeks (PK-evaluable population; Fig. 1). In the ITT population, reductions in FPG were observed at 4 weeks, with further reductions at 8 weeks that plateaued thereafter. By week 4 , when exenatide concentrations were $31 \%$ of the peak concentration, the FPG reduction $(-32.4 \pm 4.3 \mathrm{mg} / \mathrm{dL})$ was equivalent to $94 \%$ of the week 24 reduction at steady state $(-34.6 \pm 4.9 \mathrm{mg} / \mathrm{dL})$. A reduction in HbA1c of $-0.6 \% \pm 0.1 \%$ occurred at week 4 , and change in $\mathrm{HbA1c}$ plateaued by week 14 . At week 24 , the reduction in $\mathrm{HbA} 1 \mathrm{c}$ was $-1.6 \% \pm 0.1 \%$. Weight loss occurred at week $4(-0.7 \pm 0.2 \mathrm{~kg})$, and weight continued to decrease with treatment (week 24: $-2.3 \pm 0.4 \mathrm{~kg}$ ). 
Table 1 Baseline demographics and characteristics

\begin{tabular}{lll}
\hline Characteristics & \multicolumn{2}{l}{ Exenatide QW } \\
\cline { 2 - 3 } & ITT population $(\boldsymbol{n}=\mathbf{1 2 9})$ & PK-evaluable population $(\boldsymbol{n}=\mathbf{7 2})$ \\
\hline Male, $n$ (\%) & $77(59.7)$ & $45(62.5)$ \\
Age at consent, years & $56 \pm 11$ & $59 \pm 10$ \\
Race/ethnicity, $n$ (\%) & & \\
White & $81(62.8)$ & $50(69.4)$ \\
Hispanic & $37(28.7)$ & $14(19.4)$ \\
Black & $6(4.7)$ & $4(5.6)$ \\
Asian & $5(3.9)$ & $4(5.6)$ \\
Weight, $\mathrm{kg}$ & $97.0 \pm 20.7$ & $98.3 \pm 22.1$ \\
BMI, $\mathrm{kg} / \mathrm{m}^{2}$ & $33.6 \pm 5.5$ & $33.7 \pm 5.7$ \\
HbA1c, \% & $8.5 \pm 1.1$ & $8.4 \pm 1.1$ \\
FPG, $\mathrm{mg} / \mathrm{dL}$ & $173 \pm 47$ & $173 \pm 50$ \\
Duration of diabetes, years & $7 \pm 5$ & $8 \pm 6$ \\
\hline
\end{tabular}

Data are mean \pm standard deviation unless otherwise noted

$B M I$ body mass index, FPG fasting plasma glucose, $H b A l c$ glycated hemoglobin, ITT intent-to-treat, $P K$ pharmacokinetic, QW once weekly

${ }^{\mathrm{a}}$ One patient had missing baseline HbAlc; ITT population, $n=128$; PK-evaluable population, $n=71$

Exenatide QW was generally well tolerated, with no discontinuations due to nausea or vomiting [11]. Reductions in FPG and HbA1c were similar among patients with and without nausea although weight loss was numerically greater in patients with nausea (difference of $0.7 \mathrm{~kg}$; ITT population; Table 2). Nausea and vomiting decreased over the 24 -week treatment period (Fig. 2). The incidence of nausea peaked at week $6(7.2 \%)$ and that of vomiting at week 8 (2.4\%), declining thereafter.

\section{DISCUSSION}

Physicians and patients may be interested to know when novel long-acting therapies, such as the GLP-1RA exenatide QW, become effective and when maximum efficacy is achieved. This post hoc analysis demonstrated that glycemic control from exenatide QW begins before steady state concentrations are achieved, with near-maximal FPG reductions and clinically relevant $\mathrm{HbA1c}$ reductions within 4 weeks and near-maximal HbA1c reductions by 14 weeks.

In this study, measured plasma exenatide concentrations progressively increased to steady state at week 8. Similarly, reports on the PK and pharmacodynamics of exenatide QW among patients with T2D in the United States, China, and Japan showed exenatide concentrations reaching steady state after $6-8$ weeks of weekly dosing [7-10].

Consistent with multiple mechanisms of action for exenatide, multiple relationships of exenatide exposure with clinical responses were demonstrated. FPG reduction was observed 


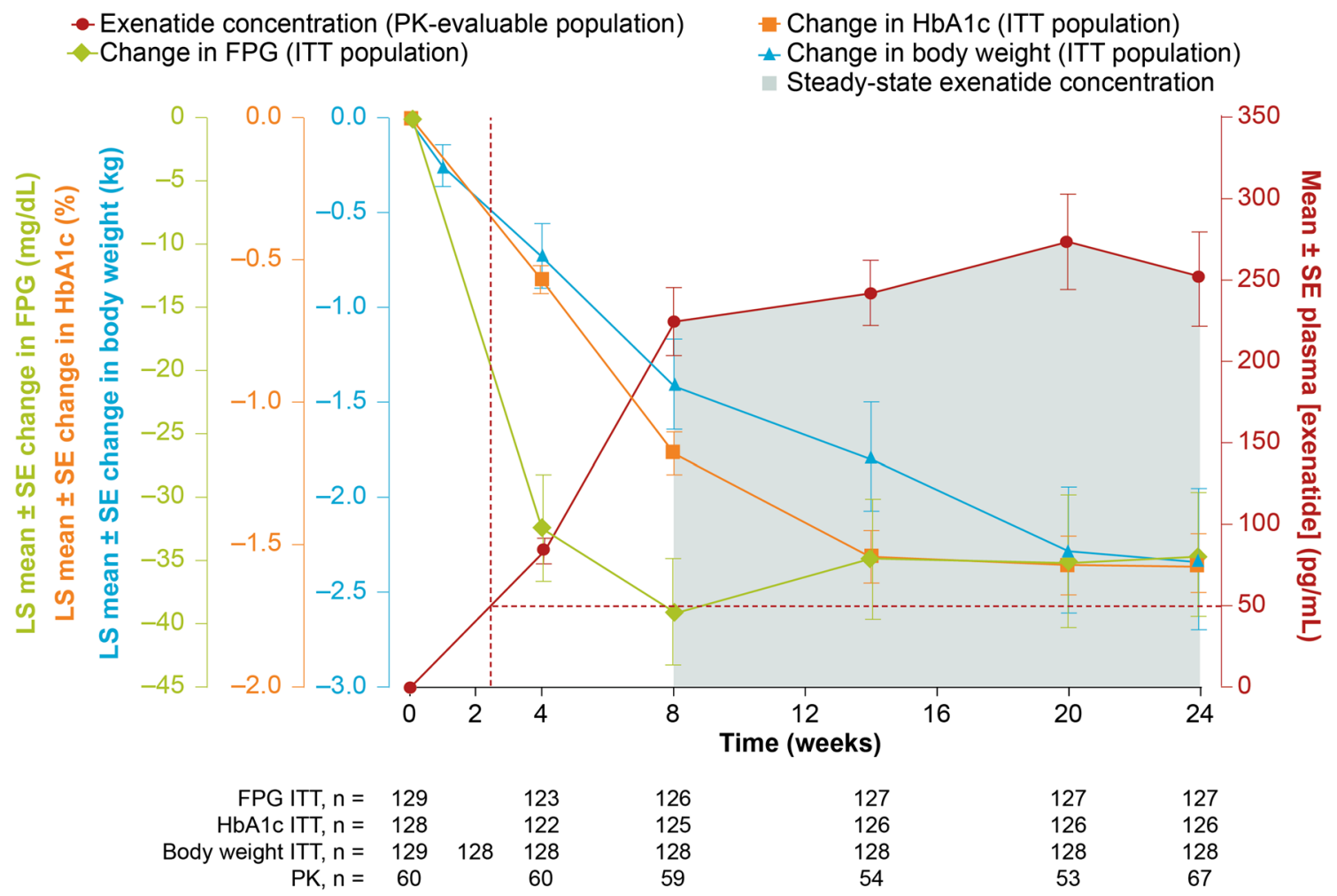

Fig. 1 Initial reductions in FPG, HbAlc, and body weight occurred before steady state exenatide concentrations were reached. Geometric mean $\pm S E$ exenatide concentrations (PK-evaluable population) and LS mean \pm SE changes from baseline (ITT population) in FPG, HbAlc, and body weight. The PK threshold for FPG lowering with exenatide QW ( $\sim 50 \mathrm{pg} / \mathrm{mL})$ is typically reached at $\sim 2$ weeks [9]. FPG fasting plasma glucose, $H b A 1 c$ glycated hemoglobin, $I T T$ intent-to-treat, $L S$ least-squares, $P K$ pharmacokinetic, $S E$ standard error

Table 2 Changes from baseline in FPG, HbAlc, and body weight in patients treated with exenatide QW with or without nausea (intent-to-treat population)

\begin{tabular}{|c|c|c|}
\hline Change in end point & Patients with nausea $(n=18)^{a}$ & Patients without nausea $(n=111)^{\mathrm{b}}$ \\
\hline $\mathrm{FPG}, \mathrm{mg} / \mathrm{dL}$ & $-32.5 \pm 44.2$ & $-31.5 \pm 50.6$ \\
\hline HbAlc, $\%^{c}$ & $-1.3 \pm 1.2$ & $-1.4 \pm 1.2$ \\
\hline Body weight, $\mathrm{kg}$ & $-3.2 \pm 3.5$ & $-2.5 \pm 3.5$ \\
\hline
\end{tabular}

Data are mean \pm standard deviation

FPG fasting plasma glucose, $H b A 1 c$ glycated hemoglobin, $Q W$ once weekly

a One patient had missing end point measures

b Four patients had missing end point measures

c Patients without nausea, baseline $n=110$

most rapidly (at week 4-the earliest time point measured), as the PK threshold for FPG lowering ( $\sim 50 \mathrm{pg} / \mathrm{mL})[12]$ is typically reached at 2 weeks
[9]. A recent post hoc analysis of pooled data from 12 clinical trials reported significant reductions in FPG, HbA1c, body weight, and 


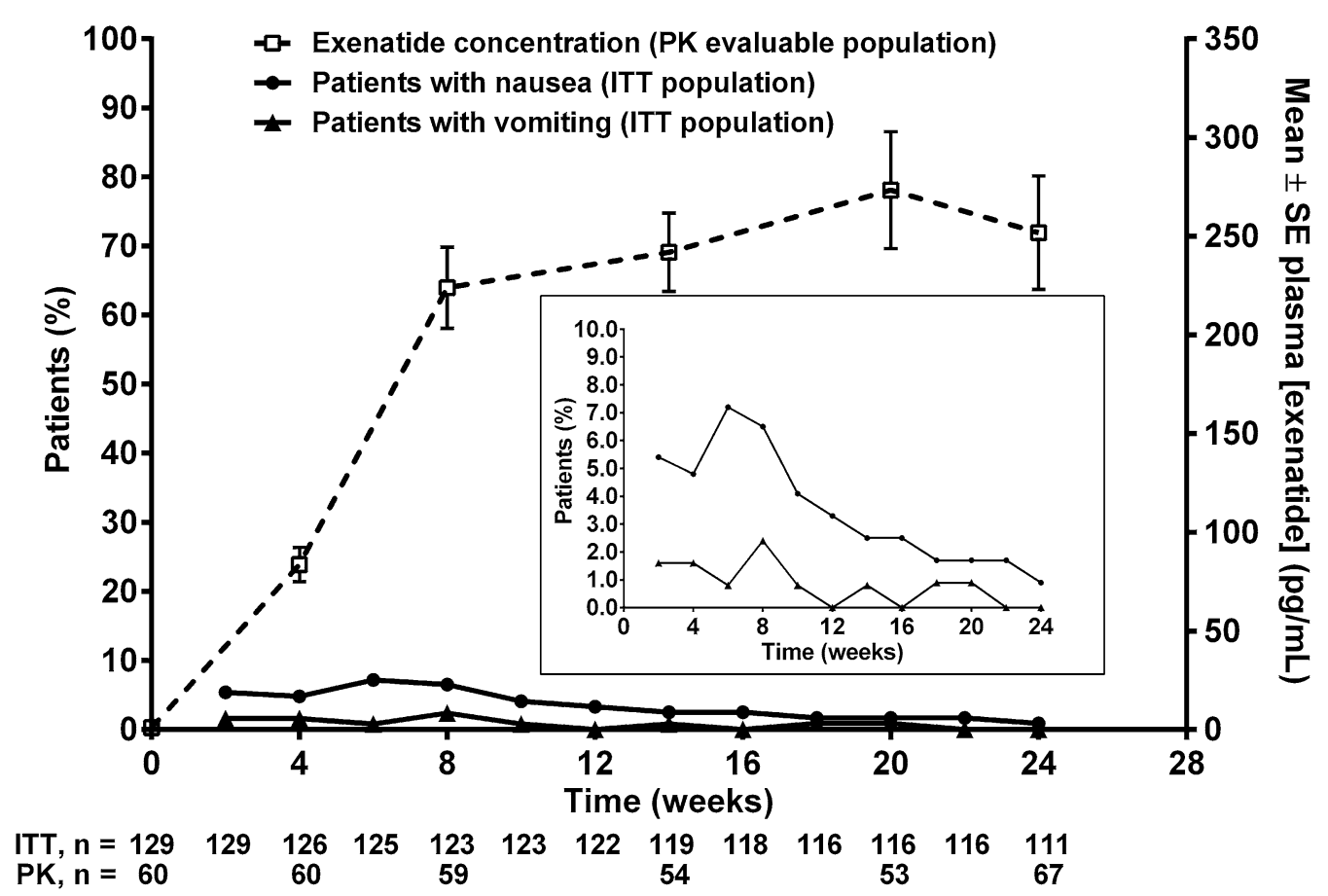

Fig. 2 Relationship of the incidences of nausea and vomiting (ITT population) with exenatide concentration (geometric mean $\pm \mathrm{SE}$; PK-evaluable population). The

blood pressure as early as 2 weeks after initiating treatment with exenatide QW, further supporting an early onset of action [13]. In this study, the time course of HbA1c change, which is more gradual than FPG because it depends on red blood cell turnover, was near maximal 14 weeks after initiation of exenatide QW. Changes in weight also occurred more slowly than effects on FPG, as the physiology of weight loss is more gradual than that of FPG reductions.

It is important for physicians and patients to understand the time course of AEs associated with a long-acting medication. As shown in this study, the majority of gastrointestinal AEs with exenatide QW occurred early in therapy, after the efficacy threshold but before steady state was reached, and declined over time as tolerance developed. A gradual increase in inset shows the incidences of nausea and vomiting on a scale from $0 \%$ to $10 \%$. ITT intent-to-treat, $P K$ pharmacokinetic, $S E$ standard error

exenatide concentrations, as occurs with exenatide QW during microsphere dissolution, has been associated with improved gastrointestinal tolerability compared with rapid dose escalation [14]. Similarly, pooled analyses of randomized controlled trials found that gastrointestinal AEs occurred less frequently with exenatide QW (providing continuous exenatide exposure) compared with exenatide BID (providing intermittent exenatide exposure), and decreased over time $[15,16]$. These analyses further support the notion that the gradual increase in exenatide concentration through the microspheres enhances gastrointestinal tolerability.

While some physicians and patients seek to avoid gastrointestinal AEs, others may believe that nausea indicates response to therapy. Our subanalysis results counter this belief, showing 
that improvements in glycemic control were similar in patients with or without nausea although weight loss may be slightly greater in patients with nausea.

Limitations of this study include the small number of patients and the post hoc nature of the analysis; in particular, there were a small number of patients with nausea in the subanalysis and it was not powered to analyze differences between patients with and without nausea. In addition, the time course of clinical responses and $\mathrm{PK}$ of exenatide QW were evaluated in different although well-matched, patient populations. This study also lacked glycemic efficacy measures at earlier (1 or 2 week) time points because they were not indicated in the protocol.

\section{CONCLUSIONS}

Although exenatide-containing microspheres deliver exenatide via a gradual release mechanism, exenatide QW resulted in prompt treatment responses once the activity threshold was reached, which occurred weeks before steady state exenatide concentrations were attained. Near-maximal improvements in FPG and clinically relevant reductions in HbA1c $(-0.6 \%)$ occurred by week 4 , followed by weight loss. The occurrence of nausea and vomiting declined over time, with tolerance developing as exenatide reached steady state concentrations.

\section{ACKNOWLEDGMENTS}

Funding for this analysis was supported by AstraZeneca. The article processing charges for this publication were funded by AstraZeneca. Amanda L. Sheldon, PhD, CMPP, of inScience Communications, Springer Healthcare
(Philadelphia, PA, USA), provided medical writing support funded by AstraZeneca. Mary Beth DeYoung, PhD, of AstraZeneca critically reviewed the manuscript. All named authors meet the International Committee of Medical Journal Editors (ICMJE) criteria for authorship for this manuscript, take responsibility for the integrity of the work as a whole, and have given final approval to the version to be published.

Disclosures. Thomas Blevins is on the speakers' bureau for AstraZeneca, Boehringer Ingelheim, Eli Lilly, Janssen, Merck, and Sanofi, and receives clinical research support from Eli Lilly, Merck, Mylan, Novo Nordisk, and Sanofi. James Ruggles and Elise Hardy are employees of AstraZeneca.

Compliance with Ethics Guidelines. This article is based on a previously conducted study and does not involve any new studies of human or animal subjects performed by any of the authors.

Open Access. This article is distributed under the terms of the Creative Commons Attribution-NonCommercial 4.0 International License (http://creativecommons.org/licenses/ by-nc/4.0/), which permits any noncommercial use, distribution, and reproduction in any medium, provided you give appropriate credit to the original author(s) and the source, provide a link to the Creative Commons license, and indicate if changes were made.

\section{REFERENCES}

1. Esposito K, Chiodini P, Maiorino MI, Bellastella G, Capuano A, Giugliano D. Glycaemic durability with dipeptidyl peptidase- 4 inhibitors in type 2 diabetes: a systematic review and meta-analysis of long-term randomised controlled trials. BMJ Open. 2014;4:e005442. 
2. Kahn SE, Haffner SM, Heise MA, Herman WH, Holman RR, Jones NP, et al. Glycemic durability of rosiglitazone, metformin, or glyburide monotherapy. N Engl J Med. 2006;355:2427-43.

3. Inzucchi SE, Bergenstal RM, Buse JB, Diamant $\mathrm{M}$, Ferrannini E, Nauck $M$, et al. Management of hyperglycemia in type 2 diabetes, 2015: a patient-centered approach: update to a position statement of the American Diabetes Association and the European Association for the Study of Diabetes. Diabetes Care. 2015;38:140-9.

4. Bhavsar S, Mudaliar S, Cherrington A. Evolution of exenatide as a diabetes therapeutic. Curr Diabetes Rev. 2013;9:161-93.

5. Wysham CH, MacConell LA, Maggs DG, Zhou M, Griffin PS, Trautmann ME. Five-year efficacy and safety data of exenatide once weekly: long-term results from the DURATION-1 randomized clinical trial. Mayo Clin Proc. 2015;90:356-65.

6. DeYoung MB, MacConell L, Sarin V, Trautmann M, Herbert P. Encapsulation of exenatide in poly-(D, L-lactide-co-glycolide) microspheres produced an investigational long-acting once-weekly formulation for type 2 diabetes. Diabetes Technol Ther. 2011;13:1145-54.

7. Iwamoto K, Nasu R, Yamamura A, Kothare PA, Mace $\mathrm{K}$, Wolka AM, et al. Safety, tolerability, pharmacokinetics, and pharmacodynamics of exenatide once weekly in Japanese patients with type 2 diabetes. Endocr J. 2009;56:951-62.

8. Kim D, MacConell L, Zhuang D, Kothare PA, Trautmann M, Fineman $M$, et al. Effects of once-weekly dosing of a long-acting release formulation of exenatide on glucose control and body weight in subjects with type 2 diabetes. Diabetes Care. 2007;30:1487-93.

9. Fineman M, Flanagan S, Taylor K, Aisporna M, Shen LZ, Mace KF, et al. Pharmacokinetics and pharmacodynamics of exenatide extended-release after single and multiple dosing. Clin Pharmacokinet. 2011;50:65-74.

10. Cui YM, Guo XH, Zhang DM, Tham LS, Tang CC, Mace $\mathrm{K}$, et al. Pharmacokinetics, safety, and tolerability of single- and multiple-dose exenatide once weekly in Chinese patients with type 2 diabetes mellitus. J Diabetes. 2013;5:127-35.

11. Blevins T, Pullman J, Malloy J, Yan P, Taylor K, Schulteis C, et al. DURATION-5: exenatide once weekly resulted in greater improvements in glycemic control compared with exenatide twice daily in patients with type 2 diabetes. J Clin Endocrinol Metab. 2011;96:1301-10.

12. Taylor K, Kim D, Nielsen LL, Aisporna M, Baron AD, Fineman MS. Day-long subcutaneous infusion of exenatide lowers glycemia in patients with type 2 diabetes. Horm Metab Res. 2005;37:627-32.

13. Trautmann M, Huang W, Ruggles J. Early pharmacodynamic effects of exenatide once weekly are independent of weight loss. Diabetes. 2015;64:A291.

14. Fineman MS, Shen LZ, Taylor K, Kim DD, Baron AD. Effectiveness of progressive dose-escalation of exenatide (exendin-4) in reducing dose-limiting side effects in subjects with type 2 diabetes. Diabetes Metab Res Rev. 2004;20:411-7.

15. Ridge T, Moretto T, MacConell L, Pencek R, Han J, Schulteis C, et al. Comparison of safety and tolerability with continuous (exenatide once weekly) or intermittent (exenatide twice daily) GLP-1 receptor agonism in patients with type 2 diabetes. Diabetes Obes Metab. 2012;14:1097-103.

16. MacConell L, Gurney K, Malloy J, Zhou M, Kolterman O. Safety and tolerability of exenatide once weekly in patients with type 2 diabetes: an integrated analysis of 4,328 patients. Diabetes Metab Syndr Obes. 2015;8:241-53. 